\title{
Le peuplement initial de Pohnpei
}

Jean-Christophe Galipaud

\section{OpenEdition}

Journals

Édition électronique

URL : http://journals.openedition.org/jso/1690

DOI : 10.4000/jso.1690

ISSN : 1760-7256

\section{Éditeur}

Société des océanistes

\section{Édition imprimée}

Date de publication : 1 juin 2001

Pagination : 49-60

ISSN : 0300-953x

\section{Référence électronique}

Jean-Christophe Galipaud, «Le peuplement initial de Pohnpei », Journal de la Société des Océanistes [En ligne], 112 | Année 2001-1, mis en ligne le 28 mai 2008, consulté le 19 avril 2019. URL : http:// journals.openedition.org/jso/1690; DOI : 10.4000/jso.1690 


\section{Le peuplement initial de Pohnpei}

par

Jean-Christophe GALIPAUD *

\section{Le peuplement ancien de la Micronésie}

La Micronésie a longtemps été considérée comme la porte d'entrée vers la Polynésie mais les dates du peuplement initial trop récentes (sauf à Guam et Palau) et surtout l'absence de poterie Lapita ont contribué à la reléguer en marge de la zone de peuplement initial de la région Pacifique. Pourtant, ces îles isolées fournissent un excellent chantier pour apprécier l'avancée du peuplement à travers les espaces maritimes océaniens. Les datations obtenues ces dix dernières années montrent que la Micronésie n'est pas restée en retrait du grand mouvement de colonisation qui a touché les îles lointaines de l'Océanie il y a 2000 ans et plus. Les données récentes confirment que la Micronésie n'a probablement jamais été une voie de pénétration vers la Polynésie, mais elle a subi des influences variées aux différentes périodes de son histoire.

Le peuplement initial des îles Mariannes (fig. 1) est estimé autour de 3600 BP à Saipan et 2700 BP à Guam, date à laquelle on trouve, dans les sites côtiers, des poteries dégraissées au sable corallien recouvertes d'une engobe rouge et décorées de motifs incisés, estampés ou d'incisions punctiformes ainsi que des objets en bénitier. La poterie des îles Mariannes est très proche stylistiquement de certaines poteries des îles Philippines et rappelle par certains aspects la poterie Lapita (Craib, 1999; Intoh, 1992 ; Spoehr, 1975).

À Yap, les dépôts les plus anciens, sous la nappe phréatique, datent de $2000 \mathrm{BP}$. Ils contiennent la même poterie dégraissée de sable corallien que l'on trouve aux îles Mariannes et aux îles
Carolines (Intoh et Leach, 1985). D'après les résultats d'une étude paléoécologique récente, les premières traces d'une influence humaine sur l'environnement dateraient de 3300 BP. Si ces dates étaient confirmées par la découverte de vestiges archéologiques de même antiquité (ce qui n'est pas le cas actuellement puisque les plus anciens sites sont datés, comme la poterie, de 2000 BP), le peuplement initial de Yap serait contemporain de celui des îles Mariannes (Dodson et Intoh, 1999).

Plus à l'est, les îles hautes de l'archipel des Carolines, Chuuk (Truk), Pohnpei (Ponape) et Kosrae, semblent avoir été visitées dès 2000 BP. Les niveaux anciens sont caractérisés, là encore, par une poterie fine, dégraissée au sable corallien plus connue sous la dénomination de Coral Sand Tempered pottery (poterie CST). Les bords, parfois incisés, suggèrent une affinité avec les poteries de la tradition plainware en Mélanésie et Polynésie occidentale (Ayres, 1990; Athens, 1990 a et b). On peut s'interroger, comme dans le cas précédemment mentionné de Yap, sur la validité de ces dates qui proviennent toutes de milieux côtiers submergés et qui sont associées à une poterie datée couramment de 2400 à 2600 BP en Mélanésie insulaire.

En Micronésie orientale, les atolls de Majuro, Bikini et Kwajalein pourraient également avoir été découverts aux alentours de 2000 BP (Streck, 1987 ; Shun et Athens, 1987). Il n’y a pas de vestiges anciens associés à ces dates en dehors de restes de poisson et peut-être de tortue, il est donc actuellement difficile d'affirmer que les charbons collectés représentent les traces d'un peuplement ancien de ces atolls plutôt que les vestiges d'une visite occasionnelle.

\footnotetext{
* Unité de recherche ADENTRO, IRD.
} 


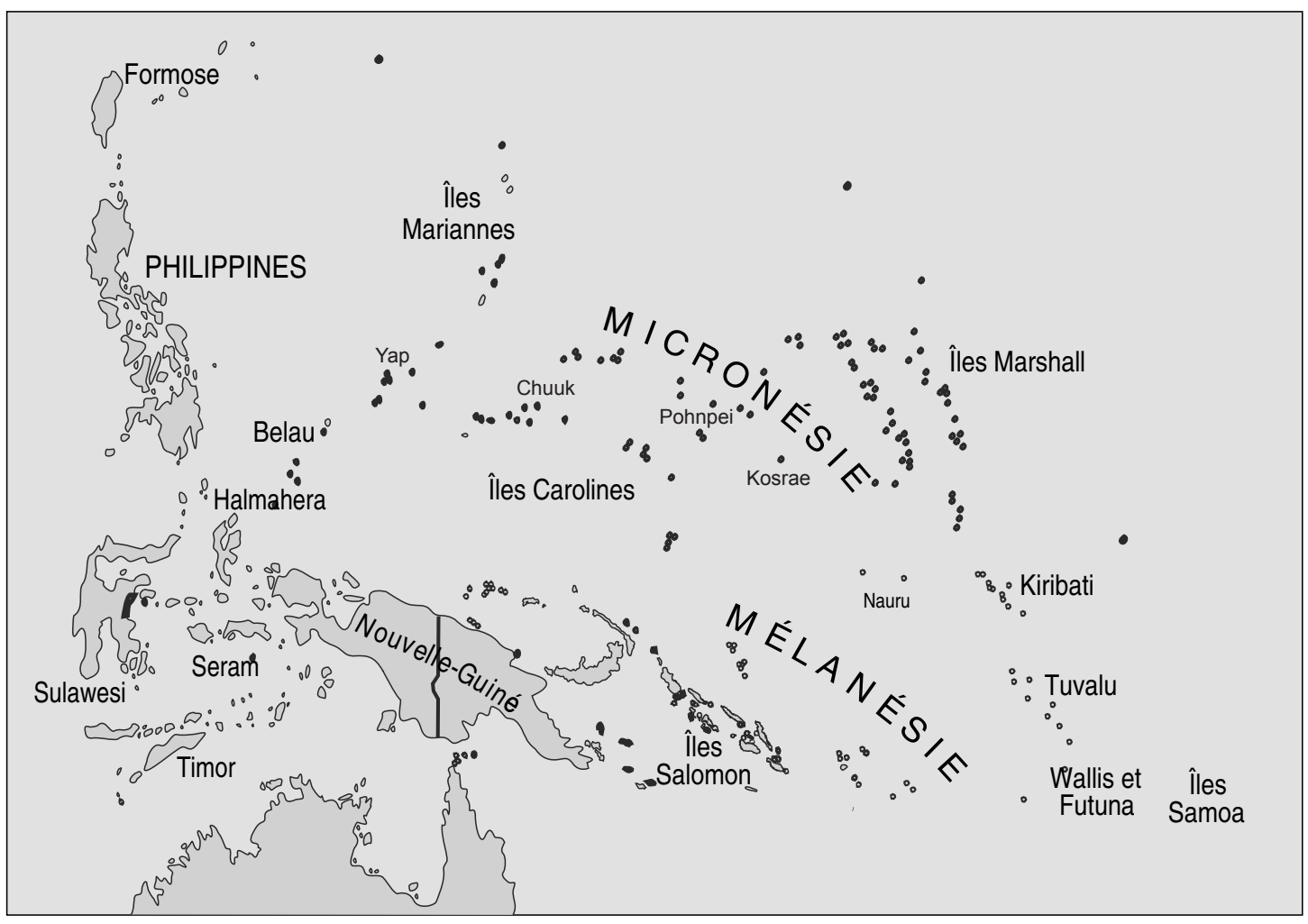

Fig. 1. - Les archipels micronésiens

Ces résultats montrent que la Micronésie n'a pas été peuplée lors d'un mouvement unique de colonisation d'ouest en est ou du sud au nord, comme l'exemple du Lapita en Mélanésie et Polynésie occidentale pouvait le suggérer. L'ancienneté probable du peuplement et les caractéristiques de la céramique dans les îles occidentales suggèrent un lien avec la culture Lapita en Mélanésie. Dans les îles Carolines, le peuplement a toutes les caractéristiques de la période plainware plus au sud à l'exception des datations, trop récentes. En Micronésie orientale, malgré quelques dates anciennes qui pourraient indiquer une découverte à la même époque qu'en Micronésie centrale, l'absence de céramique est une indication, comme en Polynésie orientale, d'une installation plus tardive dont l'origine doit être cherchée aussi bien à l'ouest qu'au sud.

Il faut néanmoins modérer ces résultats. L'absence de recherches systématiques, les transformations de ces milieux fragiles au cours des trois derniers millénaires et les remaniements importants dans les atolls pendant et après la Seconde Guerre mondiale sont autant de limites à la découverte d'une installation ancienne. Il semble que des retours vers l'ouest au cours du dernier millénaire, en particulier vers les îles hau- tes des Carolines, sont à l'origine du développement des grands complexes architecturaux (Nan Madol, Lelu) et de l'instauration d'un nouvel ordre social. Rufino Mauricio (1987 : 53 et fig. 2) a repris les textes de tradition orale relatifs à l'origine des différents groupes installés à Pohnpei et ses conclusions vont dans le sens de l'hypothèse précédente.

\section{Éléments de chronologie de Pohnpei}

Pohnpei est la plus grande des îles volcaniques de Micronésie centrale. Elle est située à quelques degrés au dessus de l'équateur, presque au centre de l'océan Pacifique, dans l'archipel des îles Carolines (fig. 1). Cette position centrale en faisait une cible accessible pour les premiers explorateurs en quête de nouvelles terres. Pohnpei est aujourd'hui connue pour abriter le complexe mégalithique de Nan Madol, un ensemble architectural de basalte, érigé entre le $\mathrm{XII}^{\mathrm{e}}$ et le XVIII ${ }^{\mathrm{e}}$ siècle sur près de cent îlots artificiels dans le lagon sud-est de l'île.

Une séquence culturelle basée sur les résultats des recherches qu'il effectua sur le site de Nan Madol et dans le nord-est de l'île (Awak) a été proposée par Ayres (1985). Il distingue six 


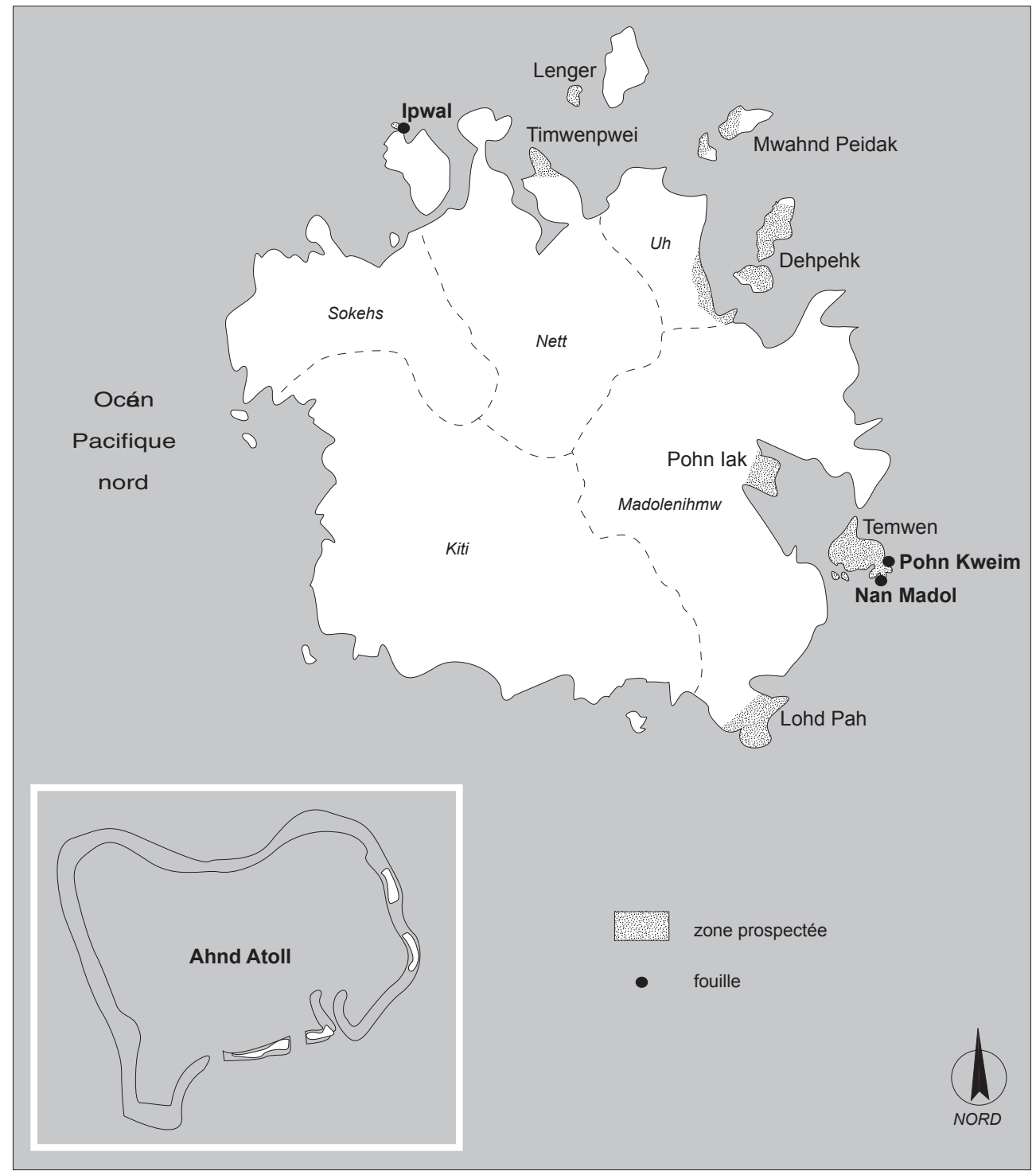

Figure 2 : Pohnpei. Localisation des zones prospectées et des fouilles. 
FIG. 2. - Pohnpei. Localisation des zones prospectées et des fouilles.

phases (tableau 1) depuis la découverte de l'île, vers $500 \mathrm{BC}$, au début de la période coloniale en 1885. Les deux phases les plus anciennes sont largement spéculatives. Elles s'appuient sur des hypothèses et n'ont pu être vérifiées à ce jour. Il n'y a aucune certitude d'un peuplement antérieur au début de notre ère à Pohnpei, même si la poterie dégraissée au sable corallien (CST) que l'on trouve en petites quantités dans les niveaux côtiers anciens de l'île Temwen, où se trouve Nan Madol, est très proche de la poterie plainware (2500-2000 BP) en Mélanésie insulaire et en Polynésie occidentale.

La date de construction du complexe mégalithique de Nan Madol, vers l'an 1000 de notre ère, est également sujette à discussions. Les pre- miers îlots artificiels pourraient dater du courant $\mathrm{du}$ premier millénaire de notre ère mais les ensembles monumentaux n'apparaissent, semble-t-il, pas avant le $\mathrm{XII}^{\mathrm{e}}$ siècle. Il est difficile de situer l'arrêt de la fabrication de la poterie locale : l'an 1500 pour Ayres ou beaucoup plus tôt si les poteries trouvées dans les remblais des constructions mégalithiques sont issues de niveaux plus anciens du bord de côte. Par comparaison, dans l'île proche de Kosrae, la poterie disparaît très rapidement vers le $\mathrm{v}^{\mathrm{e}}$ siècle de notre ère (Athens, 1990a).

Il existe donc aujourd'hui encore une grande incertitude autour de la date du peuplement initial de Pohnpei, comme d'ailleurs des autres îles de l'archipel des Carolines, et de l'évolution des 
cultures pendant le premier millénaire de notre ère. Cette situation est due au nombre limité de sites archéologiques fouillés, à la diversité des assemblages d'une île à l'autre de Micronésie et probablement également aux transformations de l'environnement côtier pendant les trois derniers millénaires.

TABLEAU 1 : Séquence culturelle dans l'île de Pohnpei (d'après Ayres 1985)

\begin{tabular}{|c|c|c|}
\hline \multicolumn{3}{|c|}{ La séquence culturelle dans l'île de Pohnpei } \\
\hline $\begin{array}{l}\text { - 1. Peuplement } \\
\text { initial }\end{array}$ & Déboisage de la forêt (Awak) et utilisation de poterie CST. & $500 \mathrm{BC} / \mathrm{AD} 0$ \\
\hline — 2. Phase Peinais & $\begin{array}{l}\text { Fondations de maisons en pierres, fosses à fermentation (arbre } \\
\text { à pain), poterie à bords incisés, îlots artificiels de Nan Madol. }\end{array}$ & AD 0 / AD 1000 \\
\hline $\begin{array}{l}\text { - 3. Phase } \\
\text { NAN MAdOL }\end{array}$ & $\begin{array}{l}\text { Expansion et formalisation du complexe de Nan Madol } \\
\text { (Empire des Saudeleur), architecture monumentale, tombes, } \\
\text { déclin de la poterie. }\end{array}$ & AD 1000/ AD 1500 \\
\hline $\begin{array}{l}\text { — 4. Phase } \\
\text { ISOHKELEKEL }\end{array}$ & $\begin{array}{l}\text { Disparition de l'empire des Saudeleur, instauration du titre } \\
\text { Namwarki, maisons de réunion (nahs). }\end{array}$ & AD $1500 /$ AD 1826 \\
\hline $\begin{array}{l}\text { 5. Début } \\
\text { du contact }\end{array}$ & $\begin{array}{l}\text { Contact avec le monde occidental, Nan Madol est toujours } \\
\text { occupé mais de façon secondaire. }\end{array}$ & AD 1826 / AD 1885 \\
\hline $\begin{array}{l}\text { - 6. Phase } \\
\text { historique }\end{array}$ & Contact avec le mode européen et gouvernement colonial. & $\mathrm{AD} 1885 / \ldots$ \\
\hline
\end{tabular}

\section{Prospection et sondages}

Athens (1980) et Ayres (1983, 1985) ont travaillé dans le sud-est de Pohnpei, sur le site de Nan Madol, ainsi qu'au nord-est, dans la baie d'Awak. J'ai choisi de démarrer mes recherches le long de cette même côte et dans les îlots proches. La prospection côtière à Pohnpei se heurte à des difficultés importantes du fait de la présence de grandes étendues de mangroves qui bloquent l'accès à la mer. Sur la côte ouest, ces mangroves peuvent atteindre 2 à $3 \mathrm{~km}$ de largeur. Elles oblitèrent toute trace d'une occupation côtière ancienne potentielle. Sur la côte est, elles sont moins abondantes et la présence de nombreux îlots rend la zone plus abordable pour la recherche de sites côtiers anciens. J'ai débuté par une prospection à la tarière et des sondages ponctuels quand cela paraissait approprié sur l'îlot Temwen et le long de la côte est de l'île (fig. 2). J'ai ensuite réalisé plusieurs sondages sur le site déjà prospecté d'Ipwal. J'avais également envisagé, en 1999, quelques sondages rapides pour évaluer les possibilités d'une occupation ancienne dans l'atoll de Ahnd, à $15 \mathrm{~km}$ au sudest de Pohnpei. Je n'ai pu prospecter cet atoll qu'en juin 2000. Les résultats de ces recherches sont détaillés ci-après, par site ou zone géographique et du nord au sud.
Ipwal

Le lieu-dit Ipwal $\left(06^{\circ} 58,825^{\prime} \mathrm{N}\right.$ et $158^{\circ} 10,847$ E) se trouve à la pointe nord-ouest de l'île Sokehs (fig. 2). La pointe basaltique ouvre sur une ancienne zone de mangrove, aujourd'hui partiellement remblayée pour permettre l'établissement d'une carrière de basalte. La zone prospectée, située entre la pointe basaltique de Sokehs et l'îlot Dolehtik, est un marais de mangrove qui débouche à l'ouest sur le lagon et se termine à l'est sur le remblai élevé par la société APSCO qui exploite la carrière.

Deux semaines d'inventaire avaient déjà été réalisées à cet endroit par l'archéologue américain Russel K. Brulotte (1993) à la demande du Service de Protection des Sites (HPO) du Département des Terres de Pohnpei, en vue de l'autorisation du remblaiement d'une partie de la mangrove. Brulotte avait mis en évidence une occupation traditionnelle de la pointe caractérisée par deux petites plates-formes basaltiques (appelées pei) dans la partie occidentale de la mangrove et d'une occupation historique marquée par des tranchées creusées pendant la dernière guerre par les soldats japonais qui occupaient l'île. La plus grande plate-forme est datée de $550 \pm 70$ BP (Beta 63386). Brulotte effectua également, dans la partie orientale de la mangrove, une série de petits sondages rapides à la 


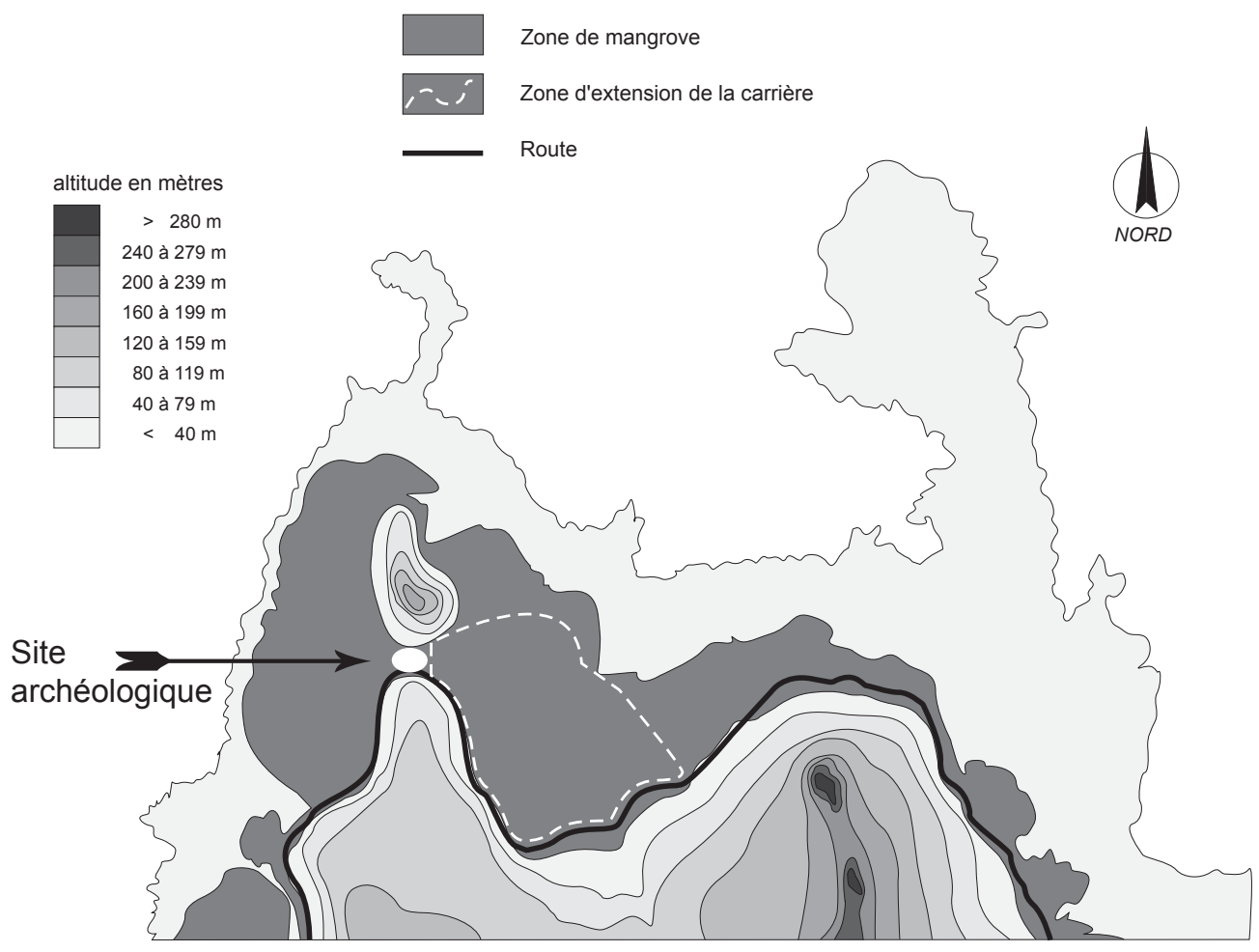

FIG. 3. - Site d'Ipwal. Emplacement du site archéologique (l'équidistance des courbes est de $40 \mathrm{~m}$ ).

pelle (46 au total) espacés les uns des autres de 10 mètres, pour tester l'éventualité d'une occupation préhistorique. Ces sondages ne livrèrent aucun matériel archéologique, à l'exception du sondage 6 dans lequel un fragment de poterie et un fragment de bracelet en tridacne furent trouvés à $120 \mathrm{~cm}$ sous la surface dans un niveau daté de $850 \pm 80$ BP (Beta 63388) ; le niveau suivant est daté de $1800 \pm 60 \mathrm{BP}$ (Beta 63389) (Brulotte, opus cité). La poterie, de type CST, pouvait indiquer une occupation plus ancienne. J'ai, pour tenter de découvrir ce niveau ancien, réalisé trois sondages le long de la côte et à peu de distance du rivage dans la mangrove (fig. 3 et 4 ).

Le sondage 1 se trouve entre la première structure mégalithique (pei) et la route d'accès à la carrière, à l'ouest d'un chemin pavé traditionnel conduisant à l'îlot Dolehtik. Les sondages 2 et 3 sont alignés à l'ouest du sondage 1 . Ces trois sondages ont été placés à environ 10 mètres de la route, ce qui les situe à quelques 15 à 25 mètres de l'aplomb de la falaise basaltique. Ils ont été respectivement fouillés jusqu'à 110,100 et $140 \mathrm{~cm}$ sous la surface malgré des problèmes techniques importants dus aux remontées d'eau salée.

La stratigraphie est quasiment identique dans les trois sondages (fig. 5). La terre argileuse en surface fait rapidement place à un terreau gras très organique, présent sur $80 \mathrm{~cm}$ environ. A 40 $\mathrm{cm}$ sous la surface, des accumulations de petits et moyens fragments de basalte marquent un épisode d'éboulement de la falaise proche (ou de redistribution des éboulis sous l'effet des vagues ou de la tectonique locale). À partir de $-80 \mathrm{~cm}$, un sable grossier d'origine marine, riche en fragments de coquilles et de corail remplace progressivement le niveau organique. Des blocs roulés de corail, de plus en plus gros, forment la base de cette stratigraphie. Cette succession d'éléments marins (à la base) puis organiques indique que la côte a subi au cours des derniers millénaires une sédimentation progressive alors que se développait le récif barrière. Cette sédimentation a fourni le terreau favorable à l'installation de la mangrove qui a peu à peu recouvert les plages littorales. La sédimentation du littoral a pu être favorisée ou accélérée par une variation soudaine du niveau de la mer.

Ces sondages ont livré, à la base, plusieurs fragments de poterie. Ces poteries sont peu roulées et l'ensemble, homogène, est caractérisé par la présence d'un sable corallien fin et abondant qui rend les tessons friables. Ces tessons appartiennent tous au groupe des poteries CST.

Les résultats des datations C14 d'Ipwal suivent l'ordre d'empilement des couches, ce qui est une bonne indication de l'absence de remaniement (tableau 2). La plus ancienne, cependant, 

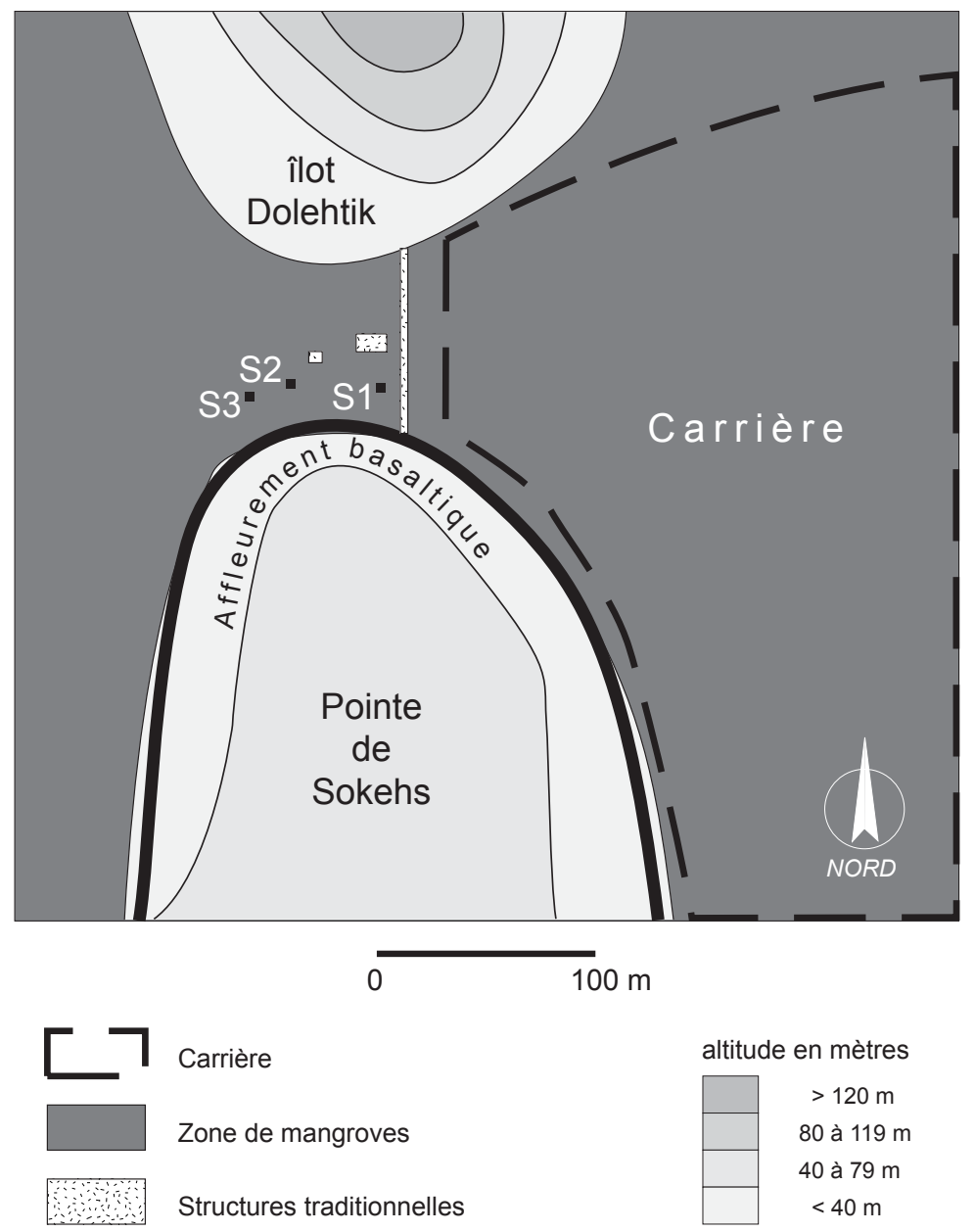

Carrière

altitude en mètres

Zone de mangroves

$$
>120 \mathrm{~m}
$$

80 à $119 \mathrm{~m}$

40 à $79 \mathrm{~m}$

Structures traditionnelles

$<40 \mathrm{~m}$

FIG. 4. - Ipwal. Plan de localisation des structures et sondages.

semble bien récente compte tenu des poteries trouvées dans le même niveau et qui sont, en Mélanésie, 300 à 400 ans plus anciennes.

Fujimoto (Fujimoto et al., 1996) situe à cette date la mise en place des niveaux anciens de mangrove et on peut se demander si le résultat du niveau $11 \mathrm{~d}$ 'Ipwal ne date pas la transformation $\mathrm{du}$ paysage côtier plutôt que l'occupation humaine du rivage. Deux des échantillons soumis pour analyse comportent des traces d'une contamination par des champignons ou algues qui, dans la mesure où les charbons se sont préservés dans un niveau submergé depuis au moins 1000 ans, ont pu affecter le taux de carbone contenu dans l'échantillon.

TABleau 2

\begin{tabular}{lllll}
\hline Code lab. & Nom du site & Âge mesuré C14 & C13/C12 & Âge conv. \\
\hline Beta-135130 & Ipwal S3, N3 & $960 \pm 110$ & $-25.0 \% 0$ & $960 \pm 110 \mathrm{BP}$ \\
Beta-135131 & Ipwal S3, N8 AMS & $1150 \pm 40$ & $-26.5 \%$. & $1130 \pm 40 \mathrm{BP}$ \\
Beta-135132 & Ipwal S3, N 11 & $1720 \pm 70$ & $-25.0 \% 0$ & $1720 \pm 70 \mathrm{BP}$ \\
Beta-135133 & Pohn Keimw, N9 AMS & $430 \pm 30$ & $-26.6 \%$ & $410 \pm 30 \mathrm{BP}$ \\
\hline
\end{tabular}




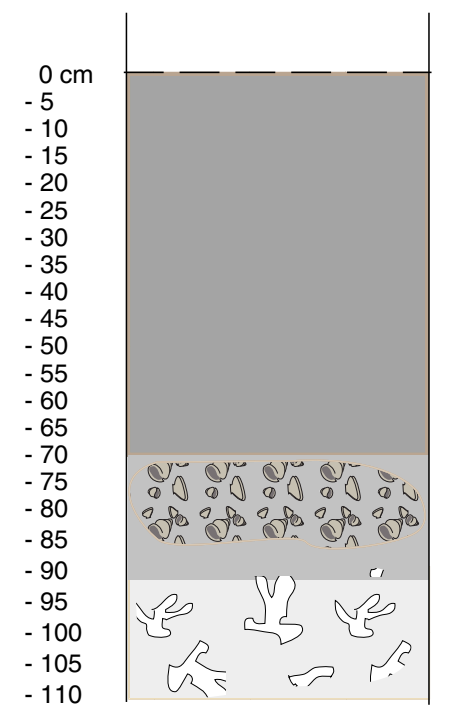

Ipwal, sondage 1

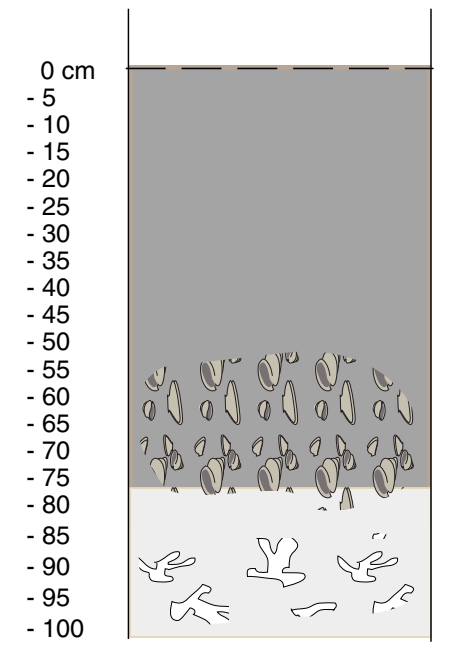

Ipwal, sondage 2

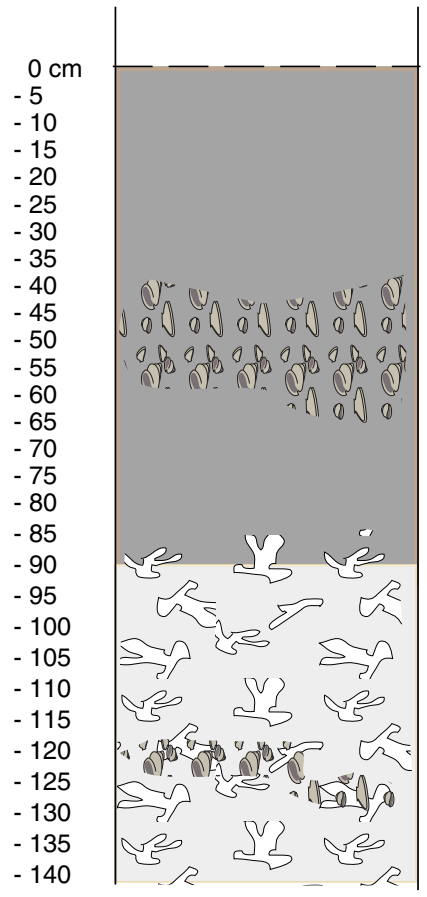

Ipwal, sondage 3

Trreau humide de mangrove

Sable brun clair

Sédiment argileux très organique, basalte

1:Dasalte

L corail

Figure 5. Coupes stratigraphiques des sondages du site d'Ipwal 
FIG. 5. - Coupes stratigraphiques des sondages du site d'Ipwal.

\section{Nan Madol et l'île Temwen}

À l'exception de deux notes rapides (Shurig, 1930 ; Yawata, 1932) suggérant qu'il existe de la poterie dans les îlots artificiels au sud de l'île Temwen, aucune poterie préhistorique ne fut réellement attestée à Pohnpei avant les travaux de Athens en 1979 sur le site de Nan Madol (Athens, 1980). Il démontra qu'il existait sur l'île Temwen des vestiges céramiques datant du début du premier millénaire de notre ère. Ayres (1983) suggéra également une date ancienne pour des poteries trouvées dans la baie d'Awak, au nord-est de l'île.

Ces deux auteurs classent la poterie en deux groupes : la 'Pohnpei plain' (poterie non décorée de Pohnpei) et la 'CST' (Calcareous Sand tempered, c'est-à-dire une poterie dégraissée au sable corallien). Athens distingue, à l'intérieur de ce second groupe, la poterie CST fine et la poterie CST grossière, suivant la taille du dégraissant.

La technique de fabrication de la poterie CST, son dégraissant entre autre, sa forme ainsi que la présence fréquente d'incisions sur le bord ont contribué à la classer dans la grande famille des plainware qui caractérisent la période suivant le Lapita dans les îles de Mélanésie et de Polynésie occidentale. Se basant sur des hypothèses linguistiques qui suggéraient que l'origine du micronésien nucléaire (Nuclear Micronesian) était située quelque part entre les îles Santa Cruz et le nord du Vanuatu, Athens (opus cité) conclut que les premiers colons de Pohnpei étaient des fabricants de poterie plainware originaires de la zone Santa Cruz / Nord Vanuatu.

Les recherches de Athens sur le site de Nan Madol ont montré que la poterie était abondante dans les niveaux profonds des îlots les plus proches de la côte. La poterie étant, pour une part au moins, bien plus ancienne que les structures, il est probable que la dispersion actuelle de la céramique est due au remaniement d'un niveau côtier lors de la construction des premiers îlots artificiels. En prospectant la côte de l'île Temwen autour du site de Nan Madol, j'ai cherché à trouver des témoins in situ de cette implantation ancienne.

La côte de l'île Temwen est rocheuse et souvent escarpée, sauf dans le sud où le site même de Nan Madol est implanté. Les endroits pouvant 


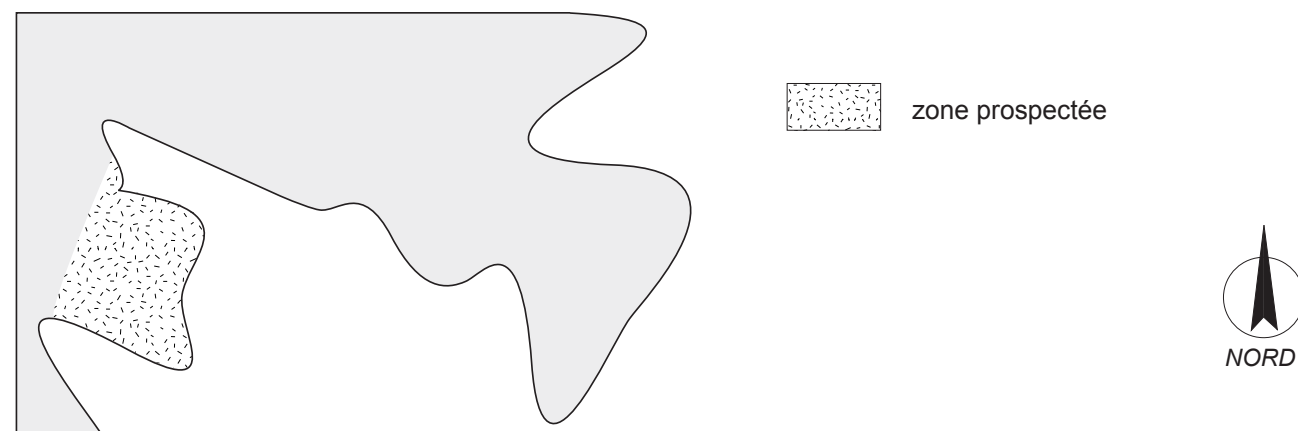

\section{Temwen}
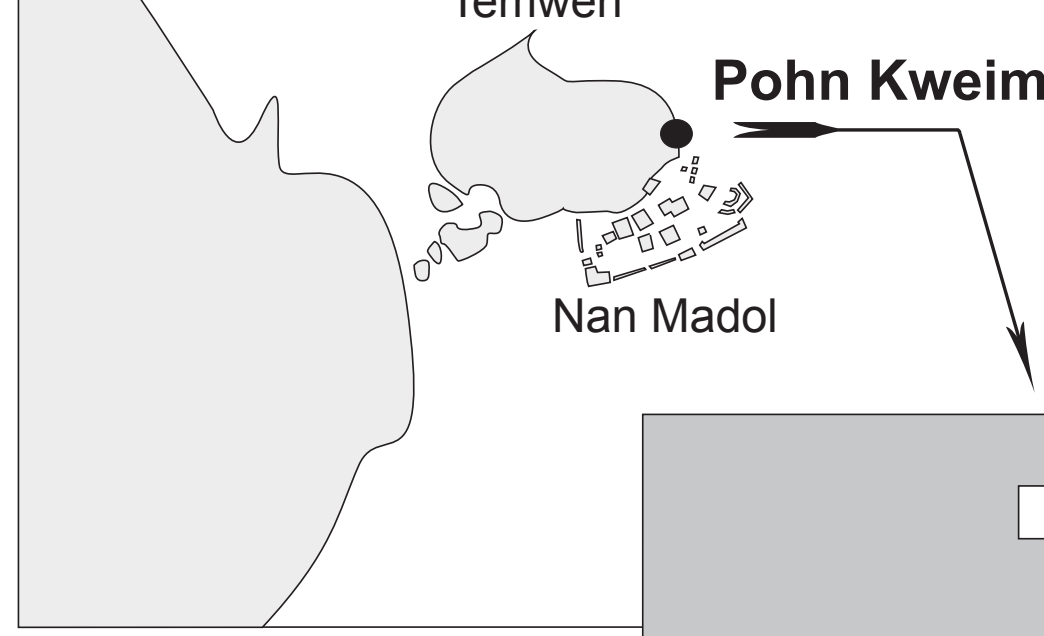

\section{Pohn Kweim}

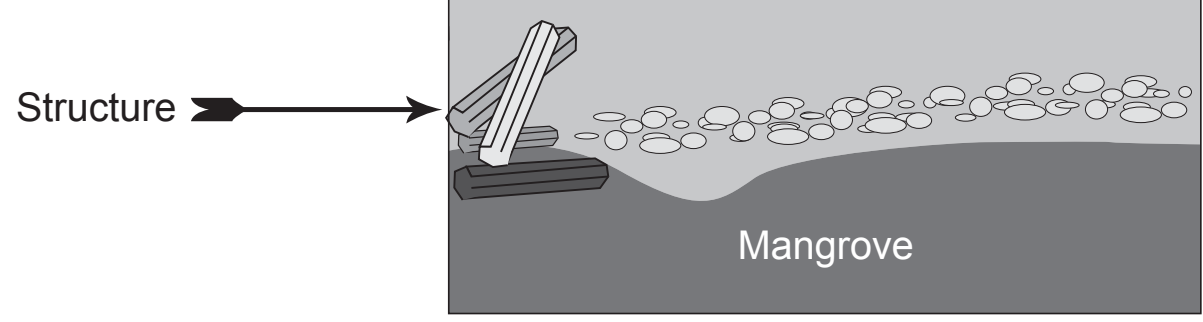

FIG. 6. - Localisation des sites et du sondage.

potentiellement abriter des niveaux anciens ne sont donc pas nombreux. Mes recherches ont porté sur la côte est, autour et au sud de l'habitation de l'actuel Nahmwarki de l'île et à la «porte» du complexe mégalithique de Nan Madol (fig. 6).

L'exploitation, pour la construction, du sable de la côte a endommagé une partie du littoral et effacé les traces potentielles d'une occupation ancienne. Les traces de l'exploitation du sable sont visibles à l'est et à l'ouest de l'île. Dans les accumulations de déchets sableux proches de la maison du Nahmwarki, j'ai trouvé plusieurs fragments de poterie et une herminette en tridacne de type IV (type B1 de Garanger).

Des sondages à la tarière dans cette zone ont été infructueux. Il y a sans doute eu une occupation ancienne dans cette partie de la côte, mais les témoins de cette occupation seront difficiles à trouver, s'ils n'ont pas été enlevés avec le sable du littoral.

Plus au sud, au lieu-dit Pohn Kweim Powe, « la porte de Nan Madol » (06 $50,941 \mathrm{~N}$ et $158^{\circ}$ $19,921 \mathrm{E})$, des tessons de poterie en surface, le long de la côte à la limite du marais, signalent une occupation ancienne potentielle. Dans ce lieu qui n'a fait récemment l'objet d'aucune culture, des plates-formes basaltiques et des pierres éparses annoncent déjà Nan Madol. Un sondage, ouvert environ dix mètres en retrait de la mangrove derrière un alignement de pierres, a été conduit jusqu'à un mètre sous la surface au milieu de blocs basaltiques et dans l'eau.

La stratigraphie (fig. 7) est simple : un sédiment fortement humifère, devenant plus gras aussitôt que le niveau de la nappe phréatique est 


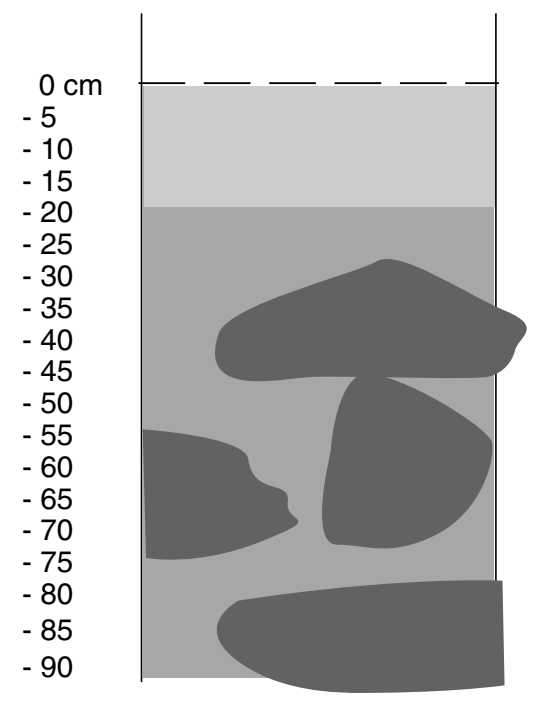

\section{Pohn Kweim, sondage 1}

Humus brun, racines et basalte

Terre argileuse brun-noir, basalte

\section{Blocs de basalte}

FIG. 7. - Stratigraphie du sondage 1, site de Pohn Kweim, île Temwen.

atteint $(-30 \mathrm{~cm})$ recouvre et emplit les interstices entre de gros blocs de basalte visiblement placés intentionnellement et recouverts plus tard par des coulées de solifluxion. À $90 \mathrm{~cm}$ sous la surface, le sondage a dû être arrêté car j'étais dans l'impossibilité d'enlever les blocs de basalte qui l'obstruaient. Les fragments de poterie sont abondants dans tous les niveaux sauf dans les niveaux 6 et 7. Dans les niveaux supérieurs, elle est érodée et les fragments sont de petite dimension alors que, dans les niveaux inférieurs, les fragments de grande dimension semblent indiquer que la poterie est en place. L'examen préliminaire des poteries montre que les deux types reconnus par Athens et Ayres sont présent, la poterie CST est néanmoins moins abondante. Un bord décoré d'incisions provient du niveau 8 .

Des charbons prélevés dans le niveau 9 , à 90 $\mathrm{cm}$ sous la surface, ont été datés de $410 \pm 30 \mathrm{BP}$ (Beta 135133). Cette datation du niveau profond de Pohn Keimw ne peut dater les poteries CST que l'on y trouve et il est probable qu'elle signale des dépôts plus récents en relation avec la construction de plates-formes basaltiques. Les élé- ments anciens (poterie CST) seraient donc ici encore en position secondaire.

\section{L'atoll de Ahnd}

Prospecté en juin 2000, cet atoll a livré de la poterie et des niveaux anciens enfouis mieux préservés qu'à Pohnpei. La description détaillée des résultats de ces travaux est en cours de publication et je n'expose ici qu'une synthèse rapide des résultats les plus marquants.

L'atoll de Ahnd se trouve à onze milles au sud-ouest de Ponape (fig. 8). Il se compose d'environ quinze îles réparties dans la région est, sud et sud-ouest d'un lagon dont le diamètre est d'environ dix miles. Les plus grandes îles sont Nikahlap, Imwansaap et Pamuk. L'intérieur du lagon est facilement accessible, même pour des gros bateaux, par une passe située au sud, entre les îles de Nikahlap et Imwansap. L'atoll faisait autrefois partie du district de Kiti. Il était occupé traditionnellement, au moins pendant la période des Saudeleur, puisque c'est d'ici que partit vers Nan Madol la flotte menée par Isokelekele qui permit de vaincre les tyrans et d'instaurer le titre de Namwarki. Pourtant les premiers blancs qui visitent ces îles, au début du XIX ${ }^{\mathrm{e}}$ siècle, pour y pêcher les bêches de mer ou la tortue, indiquent qu'elles ne sont pas habitées. Au début du $\mathrm{Xx}^{\mathrm{e}}$ siècle, la famille Nanpei, devenue propriétaire de l'atoll, y installe une exploitation de coprah et peuple l'île de gens des atolls. Cette exploitation durera jusqu'au moment de l'effondrement des cours dans les années 1960. Depuis, l'atoll est occupé sporadiquement par des pêcheurs ou des vacanciers.

Cet atoll avait déjà été prospecté en 1979 par Ayres et Mauricio qui avaient identifié un certain nombre de sites traditionnels en surface : fosses à taro (Cyrtosperma chamissonis), amas de coquillages et vestiges architecturaux de corail et basalte. Ces témoins ont été datés du courant du second millénaire de notre ère (tableau 3 ).

J'ai identifié au cours de l'année 2000 les traces d'une occupation ancienne à Pamuk (Beta145340, 2110 \pm 40 BP) et d'une occupation plus récente à Nikhalap (Beta145339, 1130 \pm 60 $\mathrm{BP}$ ). La bonne préservation des dépôts anciens et l'abondance des vestiges vont permettre de situer précisément les étapes de l'installation humaine dans ces îles et l'étude de la poterie fournira des indications précieuses sur l'origine de ce premier peuplement. Les premiers résultats suggèrent déjà que la poterie récente pourrait venir de Truk et Yap. La poterie disparaît vers l'an 1000 de notre ère. 


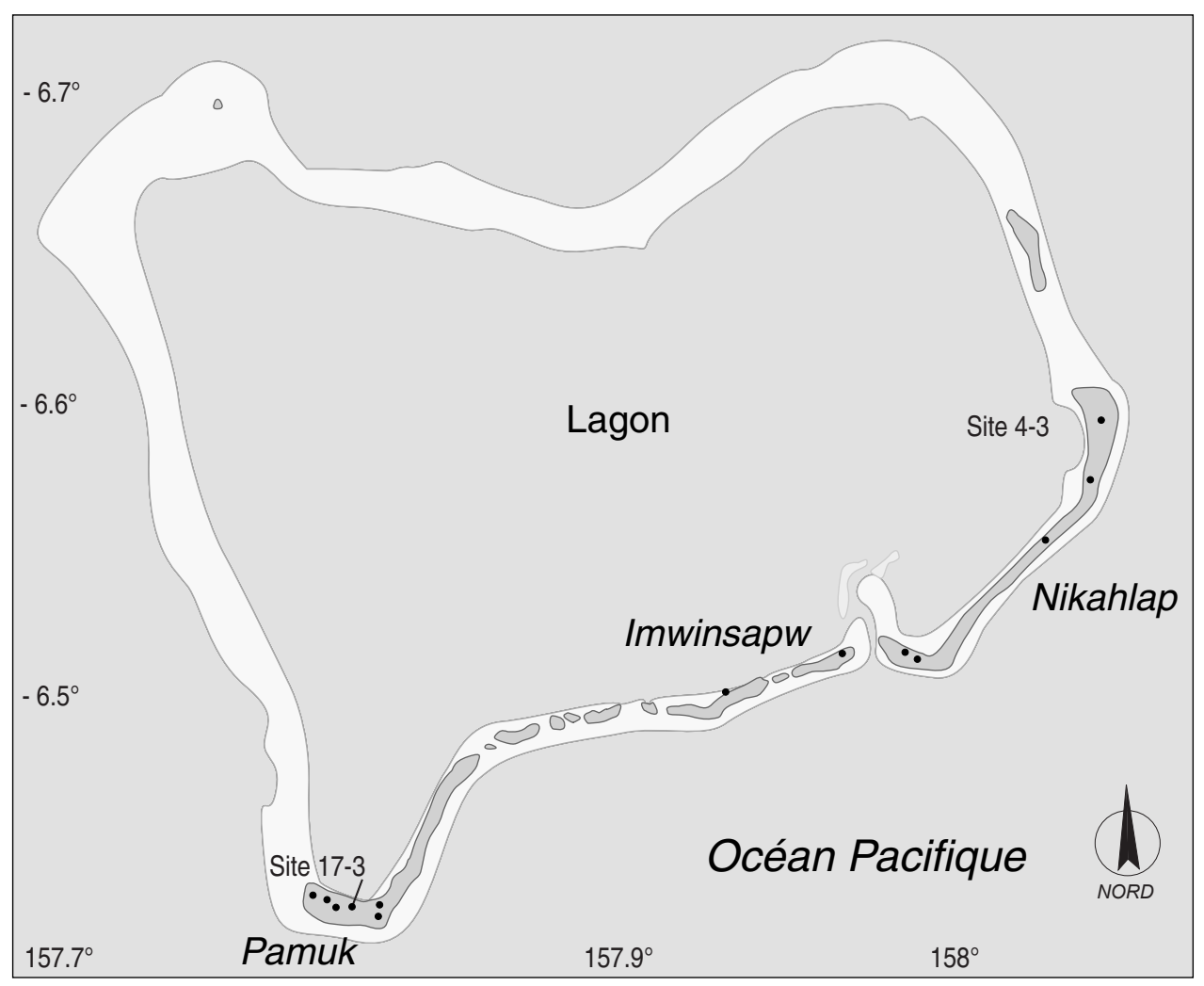

FIG. 8. - Atoll de Ahnd. Localisation des îles et des sites.

TABLEAU 3

Code Lab.

GaK 8527

GaK 8528

GaK 8529

Beta 145339

Beta 145340

Beta 145341

WK 8952
Âge mesuré C14

Datations Ayres et Mauricio

$1150 \pm 80$

$90 \pm 90$

$570 \pm 130$

$250 \pm 120$

\section{Datations Galipaud 2000}

$$
\begin{aligned}
& 1130 \pm 60 \mathrm{BP} \\
& 2110 \pm 40 \mathrm{BP} \\
& 1570 \pm 40 \mathrm{BP} \\
& 1570 \pm 60 \mathrm{BP}
\end{aligned}
$$

Site

AT 7-1

AT 4-1, $-43 \mathrm{~cm}$, top layer IV

AT $4-1$, test $1,-90 \mathrm{~cm}$

AT $4-1$, test $2,-100 \mathrm{~cm}$

Ahnd 4-3/3

Ahnd 17-3/S2/3/4

Ahnd 17/3/5

Ahnd 17/3

\section{Discussion}

Les recherches réalisées à Pohnpei en 1999 et 2000 confirment la présence d'une occupation ancienne caractérisée par la poterie CST à bords incisés mais ne permettent pas de dater plus précisément que ne l'avaient fait Athens ou Ayres l'installation humaine dans l'île.

À Ipwal, les fragments de poterie et les charbons témoignent d'une occupation ancienne de

la zone mais la stratigraphie montre sans ambiguïté que ces vestiges ont été redéposés. Les vestiges céramiques sont à l'interface des dépôts marins et côtiers, ce qui signifie qu'ils précèdent dans le temps la mise en place de la mangrove. Les charbons récoltés en association avec ces poteries peuvent être contemporains de ces dernières (déposés en même temps) ou dater le stade suivant d'érosion et de sédimentation de la zone côtière qui a favorisé la mise en place d'une 
mangrove étendue. Je favorise, en l'état actuel des recherches, la seconde hypothèse puisque des dates similaires à celle d'Ipwal situent les débuts $\mathrm{du}$ peuplement des mangroves côtières, à Pohnpei comme à Kosrae. Les mangroves d'embouchure, plus anciennes, pourraient avoir 7000 ans (Fujimoto et al., 1996).

Les poteries CST de Pohn Kweim sont également dans un contexte remanié. Elles ont probablement été apportées avec le sédiment qui a pu servir à caler les blocs des structures ou qui est arrivé naturellement par solifluxion à l'occasion d'un défrichage en amont. La seule datation de Pohn Kweim, à la base du sondage, est comparable à celle de la structure mégalithique (pei) dans la mangrove d'Ipwal. Ces deux platesformes sont probablement contemporaines; l'enfouissement de la structure de Pohn Kweim est lié à une sédimentation plus forte dans cette zone moins plate. Ce sondage n'apporte pas d'élément nouveau à la question de l'ancienneté des poteries de Nan Madol. La fraîcheur de certains des tessons trouvés en profondeur suggère qu'ils n'ont pas beaucoup migré depuis l'abandon du site mais leur position dans le sondage exclut qu'ils soient contemporains des structures. Sont-ils contemporains de structures plus anciennes comme le suggère Ayres (1000 AD) ou beaucoup plus anciens, ce que suggèrent les résultats des travaux publiés récemment sur Kosrae? Les premiers résultats obtenus dans l'atoll de Ahnd font pencher pour la seconde hypothèse. En effet, à Pamuk, la poterie CST à bord incisé est présente dans les niveaux les plus anciens (2100 BP). La poterie est toujours abondante vers $1500 \mathrm{BP}$ et encore présente, bien que moins fréquente, vers $1100 \mathrm{BP}$ à Nikahlap. Elle disparaît probablement après cette date soit au début de la phase de Nan Madol.

\section{Conclusion}

Ces travaux récents à Pohnpei et dans l'atoll de Ahnd ont confirmé la présence d'un peuplement ancien dans la zone côtière de Pohnpei et sur les atolls. La datation de ce peuplement, du début de notre ère, ne diffère pas des dates obtenues par Athens et Ayres dans différents sites de Pohnpei. Ces dates anciennes sont toujours associées à une poterie fine, souvent dégraissée au sable corallien et dont les bords sont parfois incisés (poterie CST). On rencontre ce type de poterie à la même période à Truk et plus tôt dans toute la Mélanésie (poterie plainware), mais pas dans les îles plus à l'ouest dont le peuplement initial, plus ancien, paraît associé à une poterie proche du Lapita de Mélanésie. On pouvait raisonnablement envisager l'hypothèse d'un peuplement aussi ancien à Pohnpei. Ces nouvelles recherches et les résultats précédents ne permettent pas de le démontrer même si 2000 BP semble bien récent pour la poterie CST. En terme de peuplement, il faudrait alors interpréter ces dates récentes comme l'évidence de la découverte tardive des îles de Micronésie centrale par des colons originaires de la Mélanésie ou de la Polynésie occidentale.

Ces recherches ont également confirmé que le milieu côtier à Pohnpei s'est beaucoup transformé au cours des trois derniers millénaires. Les réajustements eustatiques et iso-hydrostatiques, dont on observe les traces dans tout le Pacifique au début de notre ère, ont pu oblitérer, à Pohnpei, les traces de passage de populations plus anciennes. Les recherches vont donc se poursuivre en portant une attention particulière aux témoins de la transformation du milieu côtier au cours de cette période.

\section{REMERCIEMENTS}

Ces travaux ont été réalisés avec le soutien et la collaboration du Service de protection des sites du Département des Terres de Pohnpei dirigé par Emensio Eperiam. Rufino Mauricio m'a conseillé et aidé dans le choix des zones de prospection. Sur le terrain, Takuya Nagaoka, chercheur à l'Université d'Auckland, qui m'accompagnait lors de la mission de 1999, a assuré la liaison avec les responsables locaux et formé le personnel de fouille.

\section{RÉFÉRENCES BIBLIOGRAPHIQUES}

Athens, J. S., 1980. Pottery from Nan Madol, Ponape, Eastern Caroline Islands, Journal of the Polynesian Society 89, pp. 95-99.

_, 1990a. Kosrae pottery, clay and early settlement, Micronesica 2, pp. 171-186.

—, 1990b. Nan Madol Pottery, Pohnpei, Micronesica 2, pp. 17-32.

Ayres, W.S., 1983. Archaeology at Nan Madol, Ponape, Bulletin of the Indo-Pacific Prehistoric Association 4, pp. 135-142.

—, 1985. The archaeology of Nan Madol, Ponape, Micronesia. Preliminary report of the 1984 field research.

_, 1990. Pohnpei's position in eastern Micronesian prehistory, Micronesica 2, pp. 187-212.

BrulotTE, R. K., 1993. Ipwal Rock crushing project archaeological survey. Final Report. Kolonia. Rapport multigraphié. 33 pages. 
Craib, J.L., 1999. Colonisation of the Mariana islands: New evidence and implication for human movements in the western Pacific in J.-C. Galipaud and I Lilley (eds), The Pacific from 5000 to $2000 \mathrm{BP}$, colonisation and transformation". Édition IRD, Paris.

Dodson, J. R. et M. Intoh, 1999. Prehistory and Paleoecology of Yap, federated states of Micronesia, Quaternary International 59, pp. 17-26.

Fujimoto, K et al., 1996. Mangrove habitat formation and response to holocene sea-level changes on Kosrae island, Micronesia, Mangroves and salt marshes, 1-1. pp 47-57. SPP Academic Publishing. Amsterdam.

InTOH, M., 1992. Pottery traditions in Micronesia. In Galipaud J.C. (ed) « Lapita et peuplement. Proceedings of the lapita workshop, Orstom, Nouméa » pp 67-80.

InTOH, M. and F. LEACH, 1985. Archaeological investigations in Yap island, Micronesia - First millenium $B C$ to the present day. BAR International Series 277.

Mauricio, R., 1987. Peopling of Pohnpei island: migration, dispersal and settlement themes in clan narratives, Man and Culture in Oceania 3, pp. 47-72.
Shurig, M. 1930. Die Südsee Töpferei, Leipzig.

Shun, K. and J. S. Athens, 1987. Archaeological investigations on Kwajalein Atoll, Marshall Islands, Micronesia. Paper presented at IPPA-UOG Micronesian Archaeological Conference, Guam, September 9-12, 1987.

Shutler, R. and J. C. MARCK, 1975. On the dispersal of the austronesian horticulturalist, Archaeology and Physical Anthropology in Oceania 10, pp. 81-113.

SPOEHR, A, 1957. Marianas prehistory: archaeological survey and excavation on Saipan, Tinian and Rota, Fieldiana Anthropology 48.

STreck, Charles F., 1987. Prehistoric settlement in Eastern Micronesia : archaeology on Bikini Atoll, Republic of the Marshall Islands. Paper presented at IPPA-University of Guam Conference on Micronesian Archaeology, 9-12 September 1987

YawATA, I., 1932. Earthenware of Oceania, Zinruigaku Zassi 47(2), p. 79.

\section{Mots-clefs / Keywords}

Pohnpei, archéologie, atoll, premier peuplement, modalité d'occupation.

Pohnpei, archaeology, atoll, first colonisation, settlement pattern. 